\title{
Planets and Stellar Activity: Hide and Seek in the CoRoT-7 system
}

\author{
R. D. Haywood ${ }^{1}$, A. C. Cameron ${ }^{1}$, D. Queloz $^{2}$, S. C. C. Barros ${ }^{3}$, \\ M. Deleuil ${ }^{3}$, R. Fares ${ }^{1}$, M. Gillon ${ }^{4}$, A. Hatzes ${ }^{5}$, A. F. Lanza ${ }^{6}$, \\ C. Lovis ${ }^{2}$, C. Moutou ${ }^{3}$, F. Pepe ${ }^{2}$, D. Pollacco ${ }^{7}$, A. Santerne ${ }^{3,8}$, \\ D. Ségransan ${ }^{2}$ and Y. Unruh ${ }^{9}$ \\ ${ }^{1}$ School of Physics and Astronomy, University of St Andrews, St Andrews KY16 9SS, UK \\ email: rdh4@st-andrews.ac.uk \\ ${ }^{2}$ Observatoire de Genève, $51 \mathrm{Ch}$. des Maillettes, 1290 Sauverny, Switzerland \\ ${ }^{3}$ Aix Marseille Université, CNRS, LAM (Laboratoire d'Astrophysique de Marseille) UMR \\ 7326, 13388, Marseille, France \\ ${ }^{4}$ Institut d'Astrophysique et de Géophysique, Université de Liège, Allée du 6 août 17, Bat. \\ B5C, 4000 Liège, Belgium \\ ${ }^{5}$ Thüringer Landessternwarte, D-07778 Tautenburg, Germany \\ ${ }^{6}$ INAF-Osservatorio Astrofisico di Catania, via S. Sofia, 78 - 95123 Catania. Italy \\ ${ }^{7}$ Department of Physics, University of Warwick, Coventry CV4 7AL, UK \\ ${ }^{8}$ Centro de Astrofísica, Universidade do Porto, Rua das Estrelas, 4150-762 Porto, Portugal \\ ${ }^{9}$ Astrophysics Group, Blackett Laboratory, Imperial College London, London SW7 2AZ, UK
}

\begin{abstract}
Since the discovery of the transiting Super-Earth CoRoT-7b, several investigations have been made of the number and precise masses of planets present in the system, but they all yield different results, owing to the star's high level of activity. Radial velocity (RV) variations induced by stellar activity therefore need to be modelled and removed to allow a reliable detection of all planets in the system. We re-observed CoRoT-7 in January 2012 with both HARPS and the CoRoT satellite, so that we now have the benefit of simultaneous RV and photometric data. We fitted the off-transit variations in the CoRoT lightcurve using a harmonic decomposition similar to that implemented in Queloz et al. (2009). This fit was then used to model the stellar RV contribution, according to the methods described by Aigrain et al. (2011). This model was incorporated into a Monte Carlo Markov Chain in order to make a precise determination of the orbits of CoRoT-7b and CoRoT-7c. We also assess the evidence for the presence of one or two additional planetary companions.
\end{abstract}

Keywords. planetary systems, stellar activity, RV method

\section{Introduction}

CoRoT-7 was first brought to the attention of the scientific community in 2009, with the discovery of a transiting Super-Earth CoRoT-7b with a measured radius of $1.68 \pm 0.09$ $\mathrm{R}_{\oplus}$ (Léger et al. 2009). This discovery was followed by an intensive HARPS RV campaign (Queloz et al. 2009), in order to obtain the mass of CoRoT-7b and assess the presence of additional planets. Several analyses of these photometric and spectroscopic data were subsequently made (Bruntt et al. (2010), Lanza et al. (2010), Hatzes et al. (2010), Pont et al. (2010), Hatzes et al. (2011), Boisse et al. (2011), Ferraz-Mello et al. (2011)), but an agreement on the number and masses of planets present in the system is still to be reached. This is due to the star's high level of activity. Indeed, the presence of spots and 

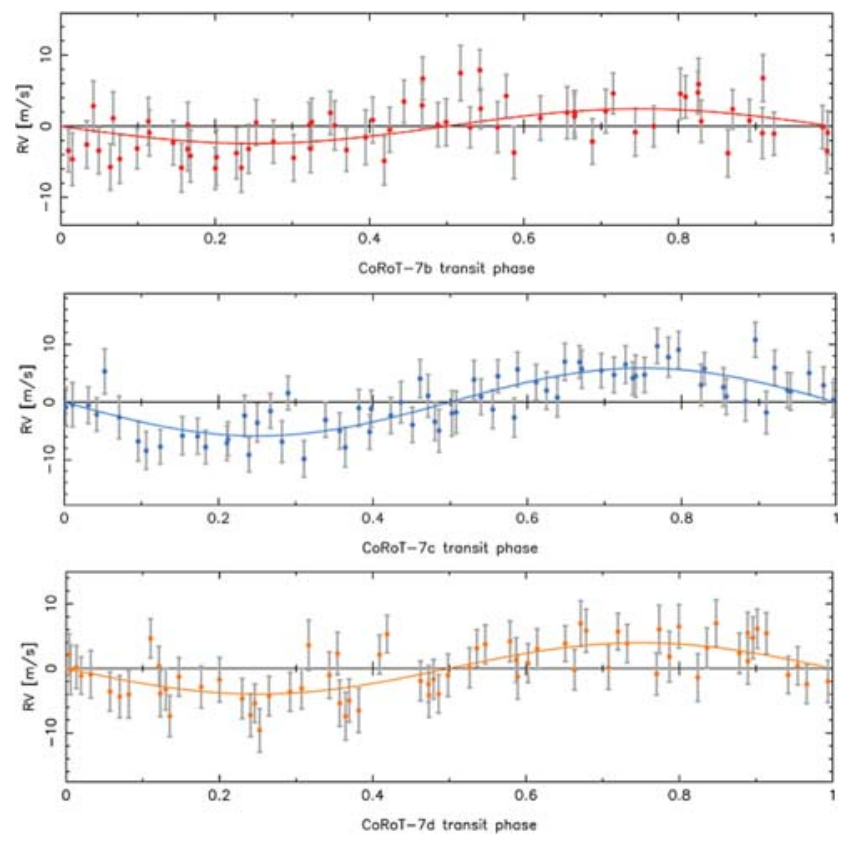

Figure 1. Phase plots of all planets, with circular orbit models overplotted.

other magnetic features on a star's surface leads to RV perturbations of the order of a few $\mathrm{m} \mathrm{s}^{-1}$ and can thus mask the RV signals produced by the orbit of low mass planets.

\section{Data and analysis}

In 2012 the CoRoT-7 system was re-observed with the CoRoT satellite (see Barros et al. (in prep.)), and with the spectrometer HARPS simultaneously. This allows us to use the star's lightcurve to model the activity-induced RV variations, according to the model presented by Aigrain et al. (2011). Details of our implementation of this method are given by Haywood et al. (submitted).

\section{Results}

We confirm the presence of three planets in the system: CoRoT-7b, with a mass of $3.38 \pm 0.86 \mathrm{M}_{\oplus}$ at an orbital period of $0.85359165 \pm 5.6 \times 10^{-7}$ day (period derived from the analysis of the 2012 transit data by Barros et al. (in prep.)), and two sub-Neptune mass planets, CoRoT-7c $\left(13.31 \pm 1.27 \mathrm{M}_{\oplus}\right)$ and CoRoT-7d $\left(11.92 \pm 2.11 \mathrm{M}_{\oplus}\right)$, at orbital periods of $3.68 \pm 0.03$ and $8.54 \pm 0.24$ days, respectively. The phase plots for each planet RV signal are shown in Figure 1.

Figure 2 shows the RV contribution of the activity models and each planet orbit. The activity-induced RV variations clearly dominate the total signal. Moreover, the residuals shown in the bottom panel of Figure 2 display some correlated noise, which may arise from incomplete modelling of activity-induced RV variations. Indeed, several physical phenomena are not accounted for in our activity model. For example, the $F F^{\prime}$ method does not consider the impact of faculae on the suppression of convective blueshift, or the RV effect of large inflows towards active regions recently found on the Sun (Gizon et al. 


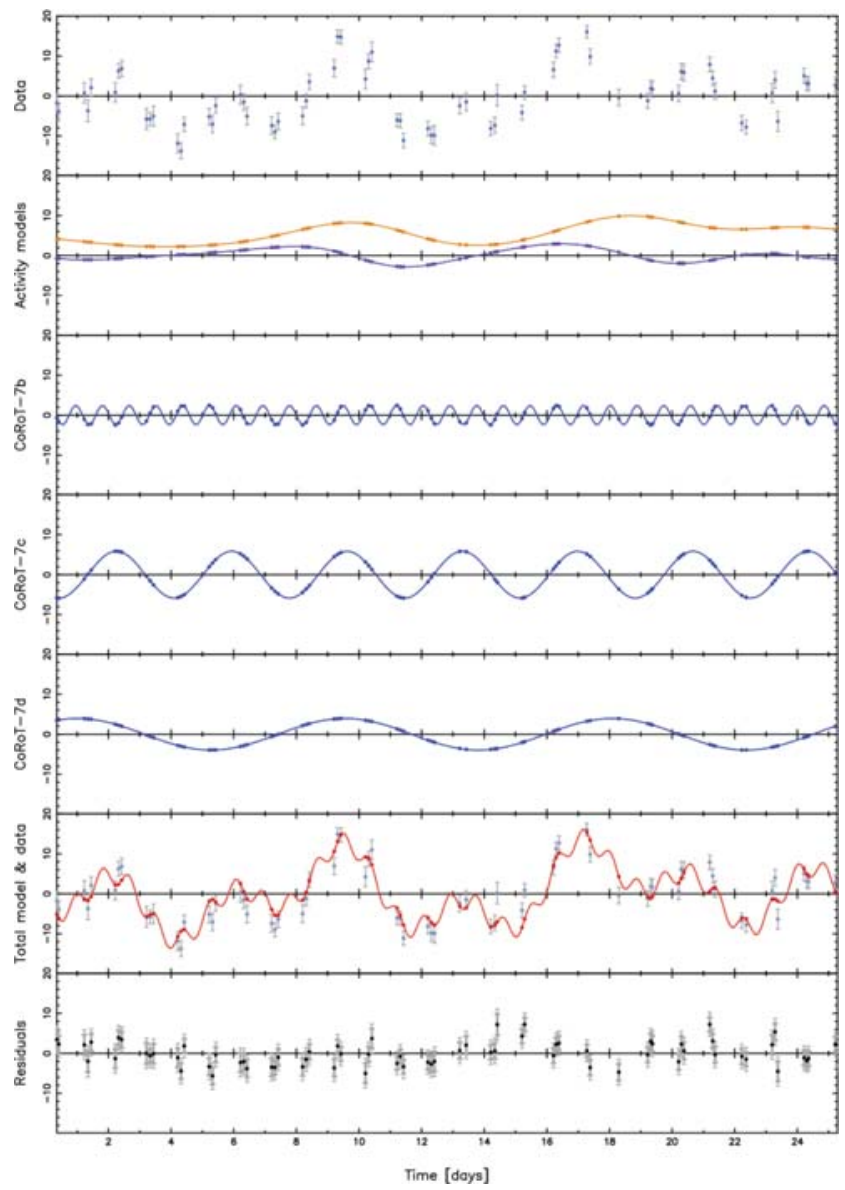

Figure 2. Time series of all the components of the total RV model. All RVs are in $\mathrm{m} \mathrm{s}^{-1}$. Second panel: activity models (model for suppression of convective blueshift (orange) and for flux blocked by starspots (purple)). Second to last panel: the total model (red) is the sum of activity and planet RVs. Subtracting it from the original data yields the residuals plotted in the bottom panel. The error bars on the residuals represent the original errors of the data (smaller bars), and the errors after an extra noise term has been added (larger bars) for comparison.

2001) - see Haywood et al. (submitted) for further discussion. In order to account for this, we add an extra noise term to the data.

\section{Conclusion}

New simultaneous photometric and spectroscopic data of the CoRoT-7 system have allowed us to model the activity-induced RV variations according to the method described by Aigrain et al. (2011). We confirm the presence of three planets, and calculate their masses. We find that the RV modulation induced by the suppression of convective blueshift and by the flux blocked by starspots are dominant contributors to the total RV of this system. Modelling the activity-induced RV is therefore essential in the case of moderately active host stars such as CoRoT-7. 


\section{References}

Aigrain, S., Pont, F., \& Zucker, S. 2011, MNRAS, 419, 4

Barros, S. C. C. et al. in prep.

Boisse, I., Bouchy, F., Hébrard, G., et al. 2011, A\& $A$, 528, A4

Bruntt, H., Deleuil, M., Fridlund, M., et al. 2010, A\&A, 519, A51

Ferraz-Mello, S., Tadeu dos Santos, M., Beaugé, C., et al. 2011, A\&A, 531, A161

Gizon, L., Duvall, T. L. Jr, \& Larsen, R. M. 2001, IAU-CP, 203, 189

Hatzes, A. P., Dvorak, R., Wuchterl, G., et al. 2010, A\&A, 520, A93

Hatzes, A. P., Fridlund, M., Nachmani, G., et al. 2011, ApJ, 743, 1

Haywood, R. D., Cameron, A. C., Queloz, D., et al. submitted, MNRAS

Lanza, A. F., Bonomo, A. S., Moutou, et al. 2010, A\& $A$, 520, A53

Léger, A., Rouan, D., Schneider, J., et al. 2009, A\&A, 506, 1

Pont, F., Aigrain, S., \& Zucker, S. 2010, MNRAS, 411, 3

Queloz, D., Bouchy, F., Moutou, C., et al. 2009, A\&A, 506, 1

\section{Discussion}

DAWSON: Is it necessary for this system to have simultaneous RV \& photometry and what are the implications for other planets orbiting (noisy?) stars?

HAYWOOD: The $F F^{\prime}$ method makes use of the lightcurve of the star to model its activityinduced RV variations. In the case of moderately active stars, active regions evolve very rapidly and can change significantly over time so it is necessary to use simultaneous photometry in order to get a reliable RV model. This need for simultaneous data is a limitation to the $F F^{\prime}$ method, but HARPS-North and Kepler have been observing several targets simultaneously so we will be able to apply the method again, and this should help us find other useful proxies to model activity induced RV.

LAGRANGE: How are the equations in the $F F^{\prime}$ method derived? How do you relate the RV signature of a spot to its photometric signature?

HAYWOOD: The equations of the $F F^{\prime}$ method are based on simple geometry. This is explained in Aigrain's paper of 2011.

DvORAK: If CoRoT-7c and 7d have eccentric orbits, then they should produce gravitational interactions and you should find a TTV (transit timing variation) - see my paper in astroph.

HAYWOOD: The analysis of the new photometric data from CoRoT is being done by Susana Barros. She did look for TTVs in the transits of CoRoT-7b but the signal to noise ratio is so small (because the planet is so small) that it is very difficult to detect any TTV with confidence. 